\title{
Glicemia de gatos obesos obtida por meio do teste de tolerância à glicose*
}

\section{Glucose of obese cats obtained through the glucose tolerance test}

\author{
Keylla Helena Nobre Pacifico Pereira, ${ }^{* *}$ Kelly Cunha Vital, ${ }^{* *}$ Luiz Eduardo Cruz dos Santos Correia, ${ }^{* *}$ \\ Bruno Neves Wanderley, ${ }^{* *}$ Karina Pessoa Oliveira, ${ }^{* *}$ Pierre Barnabé Escodro, ${ }^{* *}$ Marcia Kikuyo Notomi**
}

\begin{abstract}
Resumo
Esse trabalho avaliou a glicemia de gatos obesos por meio do teste de tolerância à glicose, a fim de identificar a hiperglicemia por resistência à insulina nesses animais. Foram avaliados 16 gatos, sendo oito com condição corpórea ideal (Grupo Controle) e oito com sobrepeso (Grupo Sobrepeso). Após jejum alimentar de 12 horas, os animais receberam glicose, via intravenosa, na dose de $0,5 \mathrm{~g} / \mathrm{kg}$ e amostras de sangue foram coletadas para aferição da glicemia no Tempo zero, após 10 (Tempo 1), 30 (Tempo 2), 60 (Tempo 3), 90 (Tempo 4) e 120 (Tempo 5) minutos. No teste de tolerância à glicose, não houve diferença significativa na comparação da glicemia entre os tempos dos dois grupos. Na comparação entre os tempos de um mesmo grupo pode-se verificar que diferentemente do Grupo Controle, não houve diferença significativa entre os Tempo 1 e Tempo 2 do Grupo Sobrepeso, sugerindo um discreto retardo na redução da concentração de glicose entre esses tempos. No estudo realizado, o teste de tolerância à glicose intravenosa apresentou-se factível, porém o período mínimo de 2 anos de obesidade foi insuficiente para a observação de alterações significativas no metabolismo da glicose em gatos obesos. Entretanto, foi possível observar o retardo na redução da concentração de glicose entre 10 e 30 minutos, o que pode estar relacionado à resistência à insulina.
\end{abstract}

Palavras-chave: diabetes, felinos, insulina, sobrepeso.

\section{Abstract}

This study evaluated by glucose tolerance test, the blood glucose of 16 cats, eight with ideal body condition (Control Group) and eight overweight cats (Overweight Group), aiming at the insulin resistance hyperglycemia identification in obese cats. After 12 hour feedings, the animals were given intravenous glucose at a dose of $0.5 \mathrm{~g} / \mathrm{kg}$ and blood samples were collected for the measurement of glycemia at time zero after 10 (Time 1), 30 (Time 2), 60 (Time 3), 90 (Time 4) and 120 (Time 5) minutes. In the glucose tolerance test, there was no significant difference in the glycemia comparison between the times of the two groups. The analysis between the times of same group, it was possible to notice that unlike Control Group, there was no significant difference between Time 1 and Time 2 of the Overweight Group, suggesting a slight delay in the glucose concentration decrease between these times, indicating a possible tendency to insulin resistance. In the study, the intravenous glucose tolerance test presented be feasible, but the minimum period of two years of obesity was insufficient for observing significant changes in glucose metabolism in obese cats. However, it was possible to observe the delay in the reduction of glucose concentration between 10 and 30 minutes, which may be related to insulin resistance.

Key words: diabetes, feline, insulin, overweight.

\section{Introdução}

A prevalência da obesidade em gatos vem aumentando e é acompanhada da elevação de diabetes mellitus na espécie. Atualmente, a obesidade é observada em $35 \%$ a $50 \%$ dos gatos e o diagnóstico de diabetes é realizado aproximadamente duas vezes mais em gatos obesos que naqueles com condição corpórea ideal (Lund et al., 2005).

A obesidade é um comprovado fator de risco para diabetes mellitus tipo 2 tanto em seres humanos, quanto em felinos domésticos e está relacionada à resistência à insulina, isto é, quando concentrações maiores de insulina são necessárias para o metabolismo da glicose (Kley et al., 2009). A resistência à insulina e a consequente hiperglicemia pós-prandial provocam lesões nas células- $\beta$ do pâncreas, ocasionando a redução na secreção de insulina, um fenômeno denominado toxicidade da glicose, sendo o mecanismo postulado como contribuinte para a progressão e manutenção de diabetes mellitus do tipo 2 (Verkest et al., 2012). Foi relatado que um aumento de $50 \%$ no índice de massa corporal (IMC) de gatos poderia levar a uma diminuição em cerca de $60 \%$ na sensibilidade à insulina (Kley et al., 2009).

Além do tratamento rígido e oneroso, a diabetes mellitus é uma enfermidade crônica com consequências severas. O diagnóstico precoce possibilita a implantação de medidas para prevenir sua evolução por meio de controle do peso e acompanhamento médico veterinário adequado (Cook, 2012).

\footnotetext{
*Recebido em 8 de setembro de 2016 e aceito em 20 de julho de 2017.

**Universidade Federal de Alagoas, Unidade Viçosa, Departamento de Clínica Veterinária, Viçosa, Alagoas, Brasil.

Autora para correspondência: Marcia Kikuyo Notomi. E-mail: marcianotomi@vicosa.ufal.br
} 
Gatos obesos apresentam resistência à insulina em tecidos periféricos (Kley et al., 2009) e podem demonstrar intolerância à glicose quando desafiados com administração desta. Apesar da possibilidade de interferência da hiperglicemia provocada por catecolaminas liberadas pela manipulação, o teste de tolerância à glicose intravenosa tem sido realizado em gatos para avaliar a eliminação de glicose (Hoenig et al., 2012).

O tempo de progressão para diabetes em gatos obesos é desconhecido (Hoenig et al., 2012). Entretanto, estudos sobre a resistência à insulina e toxicidade da glicose poderiam auxiliar no seu diagnóstico precoce e na implantação de estratégias para evitar a progressão para diabetes mellitus nessa espécie em gatos.

Dessa maneira, esse trabalho teve como objetivo, avaliar a glicemia de gatos obesos por meio do teste de tolerância à glicose, a fim de identificar a hiperglicemia por resistência à insulina nesses animais.

\section{Materiais e métodos}

O desenvolvimento do projeto foi aprovado pela Câmara de Ética em Experimentação Animal (CEUA) com protocolo ( $\left.n^{\circ} 16 / 2013\right)$ da Universidade Federal de Alagoas. O estudo foi realizado entre agosto de 2013 e julho de 2014 junto ao APEVET - Ambulatório de Pesquisa e Extensão Veterinária e Laboratório de Patologia Clínica do curso de Medicina Veterinária da Universidade Federal de Alagoas.

Foram avaliados 16 gatos adultos hígidos, considerando-se o exame clínico geral e o hemograma, provenientes de residências particulares. Os animais foram divididos em 2 grupos, o Grupo Controle, formado por 8 animais, 5 machos e 3 fêmeas, com condição corpórea ideal (Laflamme, 1997) e o Grupo Sobrepeso formado por 8 animais, 4 machos e 4 fêmeas, considerados com peso acima do ideal por um período superior a 2 anos.

Informações sobre raça, idade, condição corpórea, tipo de alimentação, frequência de alimentação, estado reprodutivo, presença de outros animais e classificação da condição corpórea na visão do proprietário, também foram registradas.

Após jejum alimentar de 12 horas, os animais receberam glicose, via intravenosa, na dose de $0,5 \mathrm{~g} / \mathrm{kg}$ e amostras de sangue foram coletadas para aferição da glicemia no Tempo zero, após 10 (Tempo 1), 30 (Tempo 2), 60 (Tempo 3), 90 (Tempo 4) e 120 (Tempo 5) minutos. A glicemia foi aferida utilizando-se um glicosímetro manual por reflectância (Accu-Chek ${ }^{\circledR}$ - Roche Diagnostics, Suíça).

No tempo zero, o sangue total foi coletado e acondicionado num tubo tipo vacutainer para separação do soro e dosagem do triglicerídeo sérico. A mensuração foi realizada através da reação Trinder (colorimétrica) utilizando-se kit específico para análise (Triglicérides Liquiform ${ }^{\circledR}$ - Labtest diagnóstica SA, Brasil).

Para as coletas das amostras de sangue, realizou-se a fixação de cateter vascular na veia cefálica. A região foi tricotomizada e submetida a uma antissepsia com álcool $70 \%$ e iodo povidine. Após as coletas das amostras, o cateter era lavado com uma solução de cloreto de sódio $0,9 \%$ heparinizada com a finalidade de evitar a obstrução por coágulos. Antes da coleta das amostras de sangue, o material no interior do cateter era desprezado a fim de evitar interferência da heparina. Após 120 minutos o cateter foi retirado.
A normalidade dos dados foi avaliada por meio do teste de Shapiro-Wilk. Os dados obtidos foram analisados quanto a sua média e desvio padrão empregando o teste de Fisher na análise de variância, e foi realizado o teste de Tukey a $5 \%$ de probabilidade para comparação dos tempos dentro do mesmo grupo e entre os grupos.

\section{Resultados e discussão}

Os 16 animais avaliados eram sem raça definida, sendo $81,25 \%$ com idade entre 2 e 4 anos e $18,75 \%$ entre 6 e 7 anos. A alimentação restrita de ração foi observada em $87,5 \%$ dos gatos e 12,5\% tinham uma alimentação mista (ração e comida caseira). Três gatos obesos $(18,75 \%)$ eram alimentados três vezes ao dia e os demais $(81,25 \%)$ animais recebiam alimentação ad libitum. Quanto ao estado reprodutivo, todos os animais do Grupo Sobrepeso e quatro do Grupo Controle eram castrados. Na visão dos proprietários do Grupo Sobrepeso, apenas 50\% consideravam seus animais com sobrepeso ou obesos. Todos os animais deste estudo conviviam com outros gatos em seus domicílios.

Os dados avaliados seguiam uma distribuição normal de acordo com o teste de normalidade de Shapiro-Wilk. Os parâmetros hematológicos dos grupos controle e sobrepeso foram comparados e não foram observadas diferenças significativas. A média da contagem de leucócitos totais apresentou elevação, ocasionada por uma neutrofilia sem desvio nos dois grupos. Os demais parâmetros encontravam-se dentro dos valores de referência para espécie.

$\mathrm{Na}$ análise da concentração de triglicerídeos séricos, observouse as médias de $387,55 \mathrm{mg} / \mathrm{dL}$ e $427,37 \mathrm{mg} / \mathrm{dL}$, para Grupo Controle e Grupo Sobrepeso, respectivamente, ambos os valores elevados para a espécie. Entretanto, não foi observada diferença significativa entre os grupos.

A Tabela 1 apresenta a comparação das médias da glicemia obtidas nos diferentes tempos entre os Grupo Controle e Grupo Sobrepeso. Não foram observadas diferenças significativas na concentração de glicose entre os dois grupos.

Tabela 1: Comparação da média da concentração de glicose $(\mathrm{mg} / \mathrm{dL})$ dos gatos dos Grupos Controle e Sobrepeso, em diferentes tempos: Tempo zero, após 10 (Tempo 1), 30 (Tempo 2), 60 (Tempo 3), 90 (Tempo 4) e 120 (Tempo 5) minutos.

\begin{tabular}{ccccc}
\hline & CONTROLE & SOBREPESO & $\mathrm{F}$ & $(\mathrm{p})$ \\
\hline Tempo 0 & 87,875 & 88,25 & 0,0017 & 0,9666 \\
Tempo 1 & 433,125 & 378,50 & 1,088 & 0,3156 \\
Tempo 2 & 298,625 & 328,88 & 0,5265 & 0,5138 \\
Tempo 3 & 240,5 & 192,50 & 1,6333 & 0,2158 \\
Tempo 4 & 121,25 & 119,13 & 0,0103 & 0,9175 \\
Tempo 5 & 88,25 & 78,63 & 1,9449 & 0,1824 \\
\hline
\end{tabular}

O resultado da análise da comparação da média glicêmica entre os tempos de um mesmo grupo está apresentado na Tabela 2. O Grupo Sobrepeso apresentou um retardo na redução da concentração da glicose entre o Tempo 1 e Tempo 2, constatado pela ausência de diferença estatisticamente significativa entre 
os dois tempos. Diferentemente, no Grupo Controle a glicemia do Tempo 3 foi significativamente menor que do Tempo 2. A persistência da hiperglicemia no Tempo 2 do Grupo Sobrepeso não foi observada nos outros tempos do grupo e após o Tempo 2.
As curvas glicêmicas do Grupo Controle e Grupo Sobrepeso estão representadas na Figura 1. Ambos os grupos apresentaram rápida elevação da glicemia e retornam a valores próximos ao Tempo 0 após 90 minutos (Tempo 4).

Tabela 2: Comparação da concentração da glicemia do grupo Controle e do grupo Sobrepeso entre os tempos (T): Tempo zero, após 10 (Tempo 1), 30 (Tempo 2), 60 (Tempo 3), 90 (Tempo 4) e 120 (Tempo 5) minutos

\begin{tabular}{|c|c|c|c|c|c|c|c|}
\hline & \multicolumn{3}{|c|}{ Controle } & & \multicolumn{3}{|c|}{ Sobrepeso } \\
\hline & Diferença & $Q$ & (p) & & Diferença & Q & (p) \\
\hline Médias $(\mathrm{T0}$ a $\mathrm{T} 1)=$ & 345,25 & 12,6089 & $<0.01$ & Médias $(\mathrm{T0}$ a $\mathrm{T} 1)=$ & 290,25 & 16,1627 & $<0.01$ \\
\hline Médias $($ T0 a T2) $=$ & 210,75 & 7,6968 & $<0.01$ & Médias $($ T0 a T2) $=$ & 240,625 & 13,3993 & $<0.01$ \\
\hline Médias $($ T0 a T3) $=$ & 152,625 & 5,574 & $<0.01$ & Médias $($ T0 a T3 $)=$ & 104,25 & 5,8052 & $<0.01$ \\
\hline Médias $($ T0 a T4) = & 33,375 & 1,2189 & Ns & Médias $($ T0 a T4) = & 30,875 & 1,7193 & Ns \\
\hline Médias $($ T0 a T5) = & 0,375 & 0,0137 & Ns & Médias ( T0 a T5) = & 9,625 & 0,536 & Ns \\
\hline Médias $(\mathrm{T} 1$ a $\mathrm{T} 2)=$ & 134,5 & 4,9121 & $<0.05$ & Médias $($ T1 a T2) $=$ & 49,625 & 2,7634 & Ns \\
\hline Médias ( T1 a T3) = & 192,625 & 7,0349 & $<0.01$ & Médias $($ T1 a T3) $=$ & 186 & 10,3575 & $<0.01$ \\
\hline Médias $(\mathrm{T} 1 \mathrm{a}$ T4) $=$ & 311,875 & 11,39 & $<0.01$ & Médias $(\mathrm{T} 1 \mathrm{a}$ T4 $)=$ & 259,375 & 14,4434 & $<0.01$ \\
\hline Médias $(\mathrm{T} 1$ a T5 $)=$ & 344,875 & 12,5952 & $<0.01$ & Médias $($ T1 a T5) $=$ & 299,875 & 16,6986 & $<0.01$ \\
\hline Médias $($ T2 a T3) $=$ & 58,125 & 2,1228 & Ns & Médias $($ T2 a T3) $=$ & 136,375 & 7,5941 & $<0.01$ \\
\hline Médias $(\mathrm{T} 2$ a T4) $=$ & 177,375 & 6,4779 & $<0.01$ & Médias $($ T2 a T4) = & 209,75 & 11,68 & $<0.01$ \\
\hline Médias $(\mathrm{T} 2$ a T5 $)=$ & 210,375 & 7,6831 & $<0.01$ & Médias $($ T2 a T5) $=$ & 250,25 & 13,9353 & $<0.01$ \\
\hline Médias $($ T3 a T4) $=$ & 119,25 & 4,3551 & $<0.05$ & Médias $($ T3 a T4 $)=$ & 73,375 & 4,0859 & Ns \\
\hline Médias $($ T3 a T5) $=$ & 152,25 & 5,5603 & $<0.01$ & Médias $($ T3 a T5) $=$ & 113,875 & 6,3412 & $<0.01$ \\
\hline Médias $($ T4 a T5) $=$ & 33 & 1,2052 & Ns & Médias (T4 a T5) = & 40,5 & 2,2553 & Ns \\
\hline
\end{tabular}

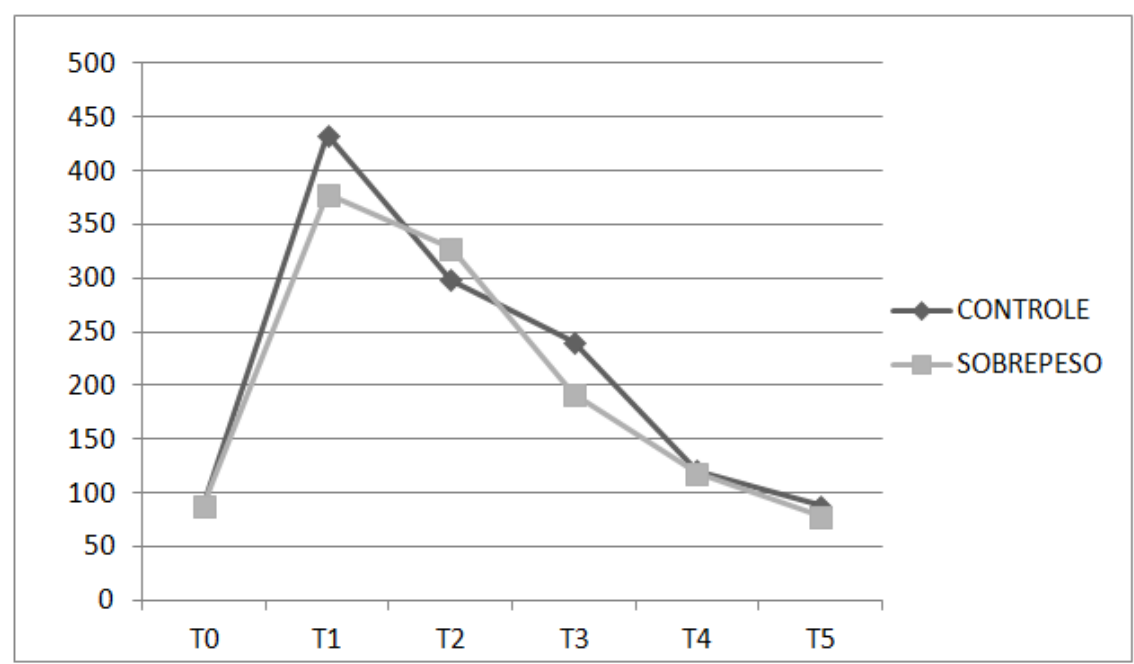

Figura 1: Curva da concentração de glicose (mg/dL) nos tempos $(\mathrm{T})$ dos Grupos Controle e de Sobrepeso ao teste de tolerância a glicose 
Nesse estudo, todos os animais pertencentes ao Grupo Sobrepeso eram castrados, concordando com Colliard et al. (2009) que incriminam a castração como um dos principais fatores de risco para a obesidade felina. Diferentes estudos relatam que gatos castrados apresentavam um maior risco ao excesso de peso ou obesidade. Esses resultados enfatizam a importância de informar aos proprietários os riscos da castração e sua associação com a obesidade (Courcier et al., 2010). Principalmente, porque muitos proprietários não reconhecem a obesidade, como nesse estudo que apenas metade dos proprietários considerava seus animais com sobrepeso.

No Grupo Controle, quatro animais eram castrados há pelo menos seis meses, no entanto mantiveram sua condição corporal normal. Isso indica que outros fatores além da castração estão envolvidos como fatores predisponentes a obesidade, como idade de castração, tipo e frequência de alimentação e convivência com outros felinos (Russel et al., 2000).

Apesar de Lund et al. (2005) referirem que a prevalência da obesidade e sobrepeso ser maior em gatos de meia-idade (entre 5 e 11 anos), neste experimento três animais do Grupo Sobrepeso estavam nessa faixa etária, enquanto os outros cinco tinham entre 2 e 4 anos e já apresentavam sobrepeso há mais de dois anos.

Durante o exame físico dos animais foi verificado que todos os gatos pertencentes ao Grupo Sobrepeso apresentavam excesso de tecido adiposo localizado no abdômen e, segundo Biourge et al. (1997), a localização da gordura corporal também pode correlacionar-se com a intolerância à glicose e a resistência à insulina, principalmente o tecido adiposo intra-abdominal que está mais associado com grave intolerância à glicose e resistência à insulina em seres humanos.

No teste de tolerância à glicose não foi observada diferença significativa na média glicêmica entre os tempos dos Grupo Controle e Grupo Sobrepeso e, em ambos grupos, o retorno aos valores iniciais de glicose tal como no Tempo 0, ocorreu como esperado para a espécie. Esta rápida recuperação está de acordo com Kley et al. (2009) que observaram que o fígado de gatos obesos ainda é sensível à insulina e que a autorregulação hepática ainda está intacta, apesar da resistência periférica à insulina. Gatos obesos compensam a resistência à insulina, mesmo em jejum e no período pós-prandial por serem capazes de manter a sensibilidade hepática à insulina e a produção de glicose endógena, o que lhes permite manter a normoglicemia (Hoenig et al., 2012).

A diferença significativa observada na comparação entre o Tempo 1 e Tempo 2 do Grupo Sobrepeso mostrou um discreto retardo na redução da concentração de glicose, isto é, uma discreta persistência da hiperglicemia, podendo sugerir uma tendência à resistência à insulina. Porém, no Tempo 4, o Grupo Sobrepeso já apresentava médias glicêmicas semelhantes ao Tempo 0 , concordando que gatos parecem compensar bem a resistência

\section{Referências}

BIOURGE, V.; NELSON, R. W.; FELDMAN, E. C. et al. Effect of weight gain and subsequent weight loss on glucose tolerance and insulin response in healthy cats. Journal of Veterinary Internal Medicine, v. 11, p.86-91, 1997. à insulina. Hoenig et al. (2012) afirmaram que isso deve ocorrer devido à diminuição da produção de glicose no fígado, mantendo as concentrações normais mesmo no período pósprandial durante vários anos, apesar da resistência à insulina. Provavelmente, a diabetes em gato só deve se desenvolver quando o fígado se torna resistente à insulina e/ou a secreção de insulina se torna demasiado baixo para superar o aumento da produção de glicose (Kley et al., 2009; Hoenig et al., 2012).

Possivelmente, os gatos com sobrepeso do experimento necessitem de um tempo mais prolongado para a observação de uma alteração mais marcante no teste de tolerância à glicose intravenosa, principalmente porque parte dos animais desse estudo eram jovens e a diabetes mellitus é uma enfermidade de animais de meia idade a idosos. Diversos mecanismos fisiopatológicos estão envolvidos na progressão da obesidade para a diabetes e podem requerer anos para se desenvolver.

A inclusão de um número maior de animais poderia auxiliar o estudo, pois nem todos os gatos obesos tornam-se diabéticos e, semelhante às outras espécies, a genética, fatores ambientais e metabólicos podem influenciar significativamente na resposta à obesidade a longo prazo (Hoenig et al., 2012).

Hoenig et al. (2012) relataram dificuldade de avaliar a concentração de glicose em gatos pela alta incidência de hiperglicemia por excitação, provocada pela liberação de catecolaminas circulantes, independente da condição corporal. Entretanto, nesse estudo, apenas um dos gatos do Grupo Controle, em que a manipulação foi mais árdua, apresentou hiperglicemia durante o Tempo 0 . Ou seja, apesar de ter sido submetido ao jejum de 12 horas, sua glicemia chegou a $112 \mathrm{mg} /$ $\mathrm{dL}$ decorrente da manipulação.

A mensuração da concentração de insulina sérica durante a realização da curva glicêmica poderia auxiliar na identificação da resistência à insulina, já que estudos realizados comprovaram com base o aumento da secreção de insulina (Verkest et al., 2012).

\section{Conclusão}

O teste de tolerância à glicose intravenosa é um factível de ser realizado, apesar da possibilidade do gato não tolerar bem a manipulação para obtenção das amostras biológicas, e ocasionalmente, ocorrer hiperglicemia.

O período mínimo de 2 anos de obesidade foi insuficiente para a observação de alterações significativas no metabolismo da glicose em gatos obesos.

Entretanto, mesmo estudando animais obesos predominantemente jovens, foi possível observar o retardo na redução da concentração de glicose entre 10 e 30 min, o que pode estar relacionado à resistência à insulina. Diante da capacidade de compensação dos gatos a resistência à insulina, alterações na curva glicêmica provavelmente só devam ocorrer após um período prolongado de obesidade.

COLLIARD, L. A.; PARAGON, B. M.; LEMUET, B. et al. Prevalence and risk factors of obesity in an urban population of healthy cats. Journal of Feline Medicine and Surgery, v.11, p.135140, 2009.

COOK, A. K. Monitoring methods for dogs and cats with diabetes mellitus. Journal of Diabetes Science and Technology, v.6, p.491, 2012. 
COURCIER, E. A.; O'HIGGINS, R.; MELLOR, D. J.; YAM, P. S. Prevalence and risk factors for feline obesity in a first opinion practice in Glasgow, Scotland. Journal of Feline Medicine and Surgery, v.12, p.746-753, 2010.

HOENIG, M; JORDAN, E. T.; GLUSHKA, J. et al. Effect of macronutrients, age, and obesity on 6- and 24-h postprandial glucose metabolism in cats. American Journal Physiology Regulative Integrative and Comparative Physiology, v.301, p.1798-1807, 2011.

KLEY, S.; HOENIG, M.; GLUSHKA, J. et al. The impact of obesity, sex and diet on hepatic glucose production in cats. American Journal of physiology, v. 296, p. 936-943, 2009.
LAFLAMME, D. Development and validation of a body condition score system for dogs. Canine Practice, v.22, p.10-15, 1997.

LUND, E. M.; ARMSTRONG, P. J.; KIRK, C. A.; KLAUSNER, J. S. Prevalence and risk factors for obesity in adult cats from private US veterinary practices. International Journal Applied Research Veterinary Medicine, v.3, p.88-96, 2005.

RUSSEL, K.; SABIN, R.; HOLT, S.; BRADLEY, R.; HARPER, E. J. Influence of feeding regimen on bodv condition in the cat. Journal of Small Animal Practice, v.41, p.1247, 2000.

VERKEST, K. R., RAND, J. S., FLEEMAN, L. M.; MORTON, J. M. Spontaneously obese dogs exhibit greater postprandial glucose, triglyceride, and insulin concentrations than lean dogs. Domestic animal endocrinology, v.42, p.103-112, 2012. 